\title{
Utilization of outpatient services in refugee settlement health facilities: a comparison by age, gender, and refugee versus host national status
}

\author{
William M Weiss ${ }^{1 *}$, Alexander Vu ${ }^{1,2}$, Hannah Tappis ${ }^{1}$, Sarah Meyer ${ }^{1}$, Christopher Haskew ${ }^{3}$ and Paul Spiegel ${ }^{3}$
}

\begin{abstract}
Background: Comparisons between refugees receiving health care in settlement-based facilities and persons living in host communities have found that refugees have better health outcomes. However, data that compares utilization of health services between refugees and the host population, and across refugee settlements, countries and regions is limited. The paper will address this information gap. The analysis in this paper uses data from the United Nations High Commissioner of Refugees (UNHCR) Health Information System (HIS).

Methods: Data about settlement populations and the use of outpatient health services were exported from the UNHCR health information system database. Tableau Desktop was used to explore the data. STATA was used for data cleaning and statistical analysis. Differences in various indicators of the use of health services by region, gender, age groups, and status (host national vs. refugee population) were analyzed for statistical significance using generalized estimating equation models that adjusted for correlated data within refugee settlements over time.
\end{abstract}

Results: Eighty-one refugee settlements were included in this study and an average population of 1.53 million refugees was receiving outpatient health services between 2008 and 2009. The crude utilization rate among refugees is 2.2 visits per person per year across all settlements. The refugee utilization rate in Asia (3.5) was higher than in Africa on average (1.8). Among refugees, females have a statistically significant higher utilization rate than males (2.4 visits per person per year vs. 2.1). The proportion of new outpatient attributable to refugees is higher than that attributable to host nationals. In the Asian settlements, only $2 \%$ outpatient visits, on average, were attributable to host community members. By contrast, in Africa, the proportion of new outpatient (OPD) visits by host nationals was $21 \%$ on average; in many Ugandan settlements, the proportion of outpatient visits attributable to host community members was higher than that for refugees. There was no statistically significant difference between the size of the male and female populations across refugee settlements. Across all settlements reporting to the UNHCR database, the percent of the refugee population that was less than five years of age is $16 \%$ on average.

Conclusions: The availability of a centralized database of health information across UNHCR-supported refugee settlements is a rich resource. The SPHERE standard for emergencies of 1-4 visits per person per year appears to be relevant for Asia in the post-emergency phase, but not for Africa. In Africa, a post-emergency standard of 1-2 visits per person per year should be considered. Although it is often assumed that the size of the female population in refugee settlements is higher than males, we found no statistically significant difference between the size of the male and female populations in refugee settlements overall. Another assumption-that the under-fives make up $20 \%$ of the settlement population during the emergency phase-does not appear to hold for the post-emergency phase; under-fives made up about $16 \%$ of refugee settlement populations.

\footnotetext{
* Correspondence: bweiss@jhsph.edu

'Department of International Health, Johns Hopkins Bloomberg School of

Public Health, 615 N. Wolfe Street, Baltimore, Maryland, 21205, USA

Full list of author information is available at the end of the article
} 


\section{Background}

The global estimate number of people who are forcibly displaced is 43.3 million at the end of 2009. Included in this population are 15.2 million refugees, of whom 10.4 million fall under mandate of the United Nations High Commissioner of Refugees [1]. Less than half of the refugees live in settlement facilities. Comparisons between refugees receiving health care in settlementbased facilities and persons living in host communities have found that refugees have better health outcomes [2]. Improved access to health services is attributed to lower neonatal mortality rates and maternal mortality among the refugees when compared to the host population in certain settings $[3,4]$. However, data comparing utilization of health services between refugees and the host population, and across refugee settlements, countries and regions is limited. The paper will compare the use of outpatient health services by age and gender, and between refugees and host populations.

The analysis in this paper uses data from the United Nations High Commissioner of Refugees (UNHCR) Health Information System (HIS). This HIS is a standardized tool used by UNHCR and its partners to public health programs delivered to populations of concern [5]. The aim has been to improve the health status of refugees and other displaced persons through evidencebased policy formulation, better management of health programs, and ultimately actions that improve refugee health. In August 2010, a total of 20 operations in Africa, Asia and Middle East and North Africa regions were reporting into the HIS using common tools and guidelines. The total population under surveillance was approximately 1.5 million refugees in 102 refugee sites and across 25 different partners.

\section{Methods}

Data about settlement populations and the use of outpatient health services were exported from the UNHCR health information system database. The data included settlement specific information about the following: month of report, total settlement population and population size by gender and age group (less than five years of age, five years of age and older). Outpatient health services data included the total number of new outpatient visits (for all causes) and a breakdown of this data by region, country, settlement, month, gender, and status (refugee versus host national). We also had data about outpatient diagnoses and a breakdown by region, country, settlement, month, age and gender. Information about use of settlement outpatient services was combined with population data to calculate utilization rates and proportions where possible. Note that population denominators were not available for information about use of settlement outpatient department (OPD) services by host nationals. Instead, we collected information on national estimates of the female and less than five years of age populations [6].

Tableau Desktop was used to explore the data [7]. STATA was used for data cleaning and statistical analysis [8]. Differences in various indicators of the use of health services by region, gender, age groups, and status (host national vs. refugee population) were analyzed for statistical significance using generalized estimating equation models that adjusted for correlated data within settlements over time.

\section{Results}

Table 1 shows the distribution of settlement reports by region and country. A significant majority of monthly settlement reports came from the African region. The number of settlements per country varied widely from one (Cameroon, Djibouti, Yemen) to 15 (Chad). In total, 81 settlements were included in this study and an average population of 1.53 million refugees was receiving outpatient health services between 2008 and 2009.

Table 1 Countries represented in the analysis by Region, Number of Camps Reporting to the UNHCR Health Information System, and Average Number of Refugees Served each Month, 2008-09*

\begin{tabular}{|c|c|c|c|}
\hline Region & $\begin{array}{l}\text { Host } \\
\text { Country }\end{array}$ & $\begin{array}{l}\text { Number of } \\
\text { Camps }\end{array}$ & $\begin{array}{l}\text { Avg Monthly Population } \\
\text { Served }\end{array}$ \\
\hline \multirow[t]{3}{*}{ Asia } & Bangladesh & 2 & 28,048 \\
\hline & Nepal & 7 & 100,525 \\
\hline & Thailand & 9 & 198,098 \\
\hline $\begin{array}{l}\text { Sub- } \\
\text { Total }\end{array}$ & & 18 & 326,671 \\
\hline \multirow[t]{13}{*}{ Africa } & Burundi & 4 & 19,546 \\
\hline & Cameroon & 1 & 3,871 \\
\hline & Chad & 15 & 257,526 \\
\hline & Djibouti & 1 & 8,688 \\
\hline & Ethiopia & 5 & 72,020 \\
\hline & Guinea & 1 & 3,341 \\
\hline & Kenya & 4 & 289,861 \\
\hline & Rwanda & 3 & 50,365 \\
\hline & Sudan & 8 & 98,714 \\
\hline & Tanzania & 5 & 198,098 \\
\hline & Uganda & 11 & 144,309 \\
\hline & Yemen & 2 & 12,115 \\
\hline & Zambia & 4 & 49,707 \\
\hline $\begin{array}{l}\text { Sub- } \\
\text { Total }\end{array}$ & & 65 & $1,246,118$ \\
\hline Total & & 81 & $1,534,832$ \\
\hline
\end{tabular}

* Countries were excluded if camps were piloting the UNHCR HIS, or where there were fewer than six monthly reports total for the two-year period for the country 
Outpatient Utilization Rates for Refugee Populations

The mean number of visits per refugee per year is displayed in Table 2. On a monthly basis, refugee settlements report the number of new outpatient visits by gender. Using these data, along with population data about females and males, a crude annualized rate of outpatient utilization was calculated along with rates for each gender. Because the UNHCR database does not include information on the size and distribution of the host populations, it was not possible to calculate utilization rates for the host national population.

\section{Crude OPD utilization rates among refugee populations}

The crude utilization rate is 2.2 visits per person per year across all settlements. The outpatient utilization rate in Asia (3.5) was higher than in Africa on average (1.8). In most settlements across countries refugees were utilizing outpatient services at the SPHERE standard of 1.0 to 4.0 visits per person per year for displaced populations in emergencies [9]. A few settlements utilization rates greater than 4.0 (e.g., Bwagiriza settlement in Burundi, Kutupalong settlement in Bangladesh, and Ban Mae Surin settlement in Thailand). And, some settlements had utilization rates lower than 1.0 (i.e., Yaroungou settlement in Chad, Madi Okollo settlement in Uganda).

\section{Gender differences in OPD utilization rates among refugee populations}

Across refugee settlements reporting to the UNHCR database, females have a statistically significant higher utilization rate than males (2.4 visits per person per year vs. 2.1). This pattern is seen in all regions. In Africa, utilizations rates for females averaged 2.0 visits per person per year compared to 1.7 for males. In Asia, female utilization rates averaged 3.8 vs. 3.2 for males. Average utilization rates for both males and females fall within the SPHERE standard of $1.0-4.0$ visits per person per year for emergencies.

\section{Proportion of New Outpatient Visits per Month by Status and Gender \\ New OPD visits per month by status}

Table 3 shows the mean proportion of new visits in a month attributable to refugees versus host nationals. The proportion of new outpatient visits to settlement health facilities attributable to refugees is higher than that attributable to host nationals. In the Asian settlements, refugees accounted for about $98 \%$ of outpatient visits. Only $2 \%$ outpatient visits, on average, were attributable to host community members. By contrast, in Africa, the proportion of new outpatient (OPD) visits by refugees and host nationals was $79 \%$ and $21 \%$, respectively. The proportion of outpatient visits attributable to host community members in Africa varied significantly from about one percent on average in Djibouti and
Rwanda to as high as $30 \%$ or greater in Sudan and Uganda. In many settlements in Uganda, the proportion of outpatient visits attributable to host community members was higher than the proportion attributable to refugees. In addition, there is a statistically significant difference in the proportion of new OPD visits attributable to host nationals between Asia and Africa (an average of $18 \%$ higher in Africa).

\section{Distribution of gender among refugee populations}

Table 4 also shows the proportion of the settlement population that is female (among refugees only). Across all settlements reporting to the UNHCR database, the percent of the refugee population that is female was about the same as the male population; there was no statistically significant difference between the size of the male and female populations in refugee settlements overall. There was some variation, however, within and between regions. Asian settlements, on average, have a slightly higher percentage of males than females, except in Bangladesh. While most of the African settlements had slightly more female refugees than males, Cameroon, Ethiopia, and Kenya have the opposite relationship.

Note that the UNHCR database does not include information on the size and distribution of the host populations living near the refugee settlements reporting to the database. For this reason, we included national estimates of the size of the female population for host countries. Asian and African countries included in the database, on average, have about the same number of males and females. There are no striking differences between the percent of refugee settlement populations that are female, and the national estimates of the percent of host country populations that are female.

\section{New OPD Visits per Month by Gender}

Table 4 shows mean proportion of new visits in a month attributable to females. In all but one country (Chad), the proportion of new OPD visits per month attributable to female refugees was higher than the female proportion of the refugee population.

In a majority of African countries, the proportion of new OPD visits per month attributable to host national females was higher than national estimates of the female population in the host country. In Asia, this happened only in Bangladesh; in the other two Asian countries, the proportion of new OPD visits per month attributable to host national females was lower than national estimates of the female population in the host country.

The proportion of new OPD visits per month attributable to female refugees was also higher than the proportion of new OPD visits attributable to females among host nationals, with the exception of Yemen and Thailand.

The proportion of new OPD visits per month attributable to women (among both refugee and host nationals) 
Table 2 Outpatient Department Utilization Rates Per Refugee Per Year by Gender, 2008-2009

\begin{tabular}{|c|c|c|c|c|c|c|c|c|}
\hline \multirow{2}{*}{ Region/Country/Camp } & & \multicolumn{2}{|c|}{ All } & \multicolumn{2}{|c|}{ Female } & \multicolumn{2}{|c|}{ Male } & \multirow[t]{2}{*}{ M v. F p Value* } \\
\hline & & $\underset{*}{\text { Rate/Year }}$ & $95 \% \mathrm{Cl}^{*}$ & Rate/Year * & $95 \% \mathrm{Cl}^{*}$ & Rate/Year * & $95 \% \mathrm{Cl}^{*}$ & \\
\hline Africa & & 1.8 & $1.7,2.0$ & 2.0 & $1.8,2.1$ & 1.7 & $1.5,1.8$ & $<0.001$ \\
\hline Burundi & & 4.0 & $3.0,5.1$ & 4.2 & $3.1,5.4$ & 3.8 & $2.8,4.8$ & $<0.001$ \\
\hline Bwagiriza & & 8.4 & $6.1,10.7$ & 8.8 & $6.2,11.4$ & 8.0 & $6.2,9.8$ & \\
\hline Gasorwe & & 2.5 & $2.3,2.7$ & 2.6 & $2.3,2.8$ & 2.4 & $2.1,2.6$ & \\
\hline Gihinga & & 4.3 & $3.7,4.9$ & 4.7 & $4.1,5.4$ & 3.8 & $3.2,4.3$ & \\
\hline Musasa & & 3.7 & $3.0,4.4$ & 3.8 & $3.0,4.6$ & 3.6 & $3.0,4.2$ & \\
\hline Cameroon & Langui & 3.9 & $3.2,4.5$ & 4.1 & $3.4,4.8$ & 3.7 & $3.0,4.3$ & $<0.05$ \\
\hline Chad & & 1.4 & $1.2,1.6$ & 1.6 & $1.2,2.1$ & 1.4 & $1.3,1.6$ & \\
\hline Amboko & & 1.3 & $0.9,1.6$ & 1.2 & $0.8,1.6$ & 1.4 & $0.8,2.0$ & \\
\hline Amnabak & & 0.8 & $0.6,1.0$ & 0.7 & $0.6,0.9$ & 1.0 & $0.7,1.2$ & \\
\hline Bredjing & & 1.3 & $1.2,1.4$ & 1.2 & $1.1,1.4$ & 1.3 & $1.2,1.4$ & \\
\hline Djabal & & 1.9 & $1.7,2.1$ & 1.9 & $1.7,2.1$ & 2.0 & $1.8,2.1$ & \\
\hline Dosseye & & 2.3 & $2.0,2.6$ & 2.5 & $2.2,2.8$ & 2.1 & $1.8,2.4$ & \\
\hline Farchana & & 1.0 & $0.8,1.2$ & 0.9 & $0.7,1.1$ & 1.2 & $1.0,1.5$ & \\
\hline Gaga & & 1.1 & $0.9,1.3$ & 1.1 & $0.9,1.3$ & 1.1 & $0.9,1.3$ & \\
\hline Gondje & & 0.9 & $0.6,1.2$ & 0.9 & $0.6,1.2$ & 0.9 & $0.6,1.2$ & \\
\hline Goz Amer & & 2.0 & $1.7,2.2$ & 2.0 & $1.7,2.2$ & 2.0 & $1.8,2.2$ & \\
\hline Kounoungou & & 1.1 & $0.9,1.2$ & 1.0 & $0.9,1.2$ & 1.1 & $1.0,1.3$ & \\
\hline Mile & & 1.0 & $0.9,1.1$ & 1.0 & $0.9,1.2$ & 1.0 & $0.9,1.1$ & \\
\hline Moula & & 3.5 & $3.3,3.6$ & 3.7 & $3.6,3.9$ & 3.2 & $3.0,3.4$ & \\
\hline Oure Cassoni & & 1.3 & $1.1,1.4$ & 1.2 & $1.1,1.3$ & 1.3 & $1.2,1.5$ & \\
\hline Treguine & & 1.8 & $1.6,2.1$ & 4.7 & $-0.7,10.0$ & 1.9 & $1.7,2,2$ & \\
\hline Yaroungou & & 0.7 & $0.5,0.8$ & 0.7 & $0.5,1.0$ & 0.6 & $0.5,0.7$ & \\
\hline Djibouti & Ali Adde & 2.8 & $2.3,3.2$ & 3.1 & $2.6,3.6$ & 2.5 & $2.1,2.9$ & $<0.001$ \\
\hline Ethiopia & & 1.7 & $1.2,2.1$ & 2.0 & $1.4,2.5$ & 1.5 & $1.1,1.9$ & $<0.001$ \\
\hline Awbarre & & 0.9 & $0.8,1.1$ & 1.1 & $0.9,1.3$ & 0.8 & $0.7,0.9$ & \\
\hline Fugnido & & 1.3 & $1.0,1.5$ & 1.3 & $1.1,1.6$ & 1.2 & $0.9,1.5$ & \\
\hline Kebribeyah & & 1.7 & $1.5,1.9$ & 1.9 & $1.7,2.0$ & 1.6 & $1.4,1.7$ & \\
\hline Sherkole & & 1.9 & $1.0,2.8$ & 2.1 & $1.2,3.0$ & 1.7 & $0.9,2.6$ & \\
\hline Shimelba & & 2.7 & $1.3,4.1$ & 3.6 & $2.3,4.8$ & 2.3 & $1.0,3.6$ & \\
\hline Guinea & Kouankan II & 3.2 & $2.3,4.0$ & 3.4 & $2.4,4.4$ & 2.9 & $2.2,3.6$ & $<0.05$ \\
\hline Kenya & & 1.4 & $1.3,1.6$ & 1.5 & $1.3,1.7$ & 1.4 & $1.2,1.5$ & $<0.001$ \\
\hline Dagahaley & & 1.3 & $1.2,1.4$ & 1.4 & $1.2,1.5$ & 1.2 & $1.1,1.4$ & \\
\hline Hagadera & & 1.1 & $1.1,1.2$ & 1.2 & $1.1,1.3$ & 1.1 & $1.0,1.1$ & \\
\hline Ifo & & 1.3 & $1.2,1.4$ & 1.4 & $1.3,1.5$ & 1.3 & $1.1,1.5$ & \\
\hline Kakuma & & 1.9 & $1.6,2.1$ & 2.0 & $1.8,2.3$ & 1.8 & $1.5,2.0$ & \\
\hline Rwanda & & 1.7 & $1.1,2.4$ & 1.7 & $1.1,2.3$ & 1.7 & $1.0,2.4$ & \\
\hline Gihembe & & 1.3 & $1.0,1.6$ & 1.3 & $1.0,1.6$ & 1.3 & $1.0,1.6$ & \\
\hline Kiziba & & 1.0 & $0.9,1.2$ & 1.1 & $1.0,1.2$ & 1.0 & $0.8,1.1$ & \\
\hline Nyabiheke & & 3.0 & $2.1,4.0$ & 2.9 & $2.1,3.8$ & 3.2 & $2.1,4.4$ & \\
\hline Sudan & & 2.1 & $1.6,2.6$ & 2.4 & $1.9,2.8$ & 1.8 & $1.4,2.3$ & $<0.001$ \\
\hline Abuda & & 2.7 & $2.3,3.0$ & 3.3 & $2.8,3.9$ & 3.2 & $2.1,4.4$ & \\
\hline Fau 5 & & 4.5 & $3.5,5.5$ & 4.5 & $3.6,5.5$ & 4.3 & $3.3,5.3$ & \\
\hline Girba & & 1.7 & $1.6,1.8$ & 1.9 & $1.8,2.1$ & 1.4 & $1.3,1.5$ & \\
\hline Kilo 26 & & 1.8 & $1.5,2.0$ & 2.2 & $1.9,2.5$ & 1.5 & $1.3,1.6$ & \\
\hline Shagarab | || |I| & & 1.8 & $1.6,2.0$ & 2.2 & $1.8,2.6$ & 1.5 & $1.4,1.6$ & \\
\hline Suki & & 2.6 & $2.3,2.8$ & 3.0 & $2.7,3.2$ & 2.2 & $2.0,2.5$ & \\
\hline Um Gargour & & 0.9 & $0.7,1.1$ & 1.2 & $0.8,1.5$ & 0.8 & $0.6,1.0$ & \\
\hline Wad Sharifey & & 1.3 & $1.1,1.5$ & 1.3 & $1.1,1.5$ & 1.2 & $1.0,1.5$ & \\
\hline
\end{tabular}


Table 2 Outpatient Department Utilization Rates Per Refugee Per Year by Gender, 2008-2009 (Continued)

\begin{tabular}{|c|c|c|c|c|c|c|c|c|}
\hline Tanzania & & 2.6 & $2.2,3.0$ & 2.7 & $2.3,3.2$ & 2.4 & $2.1,2.7$ & $<0.001$ \\
\hline Lugufu & & 2.2 & $1.9,2.5$ & 2.1 & $1.8,2.5$ & 2.2 & $1.9,2.6$ & \\
\hline Lukole & & 3.3 & $2.3,4.2$ & 3.7 & $2.6,4.9$ & 2.9 & $2.1,3.6$ & \\
\hline Mtabila & & 2.8 & $2.5,3.1$ & 3.1 & $2.7,3.4$ & 2.5 & $2.3,2.8$ & \\
\hline Nduta & & 3.4 & $2.3,4.4$ & 3.6 & $2.5,4.8$ & 3.1 & $2.1,4.1$ & \\
\hline Nyarugusu & & 1.9 & $1.4,2.4$ & 1.9 & $1.4,2.5$ & 1.9 & $1.3,2.4$ & \\
\hline Uganda & & 1.2 & $1.0,1.4$ & 1.4 & $1.2,1.6$ & 1.0 & $0.9,1.2$ & $<0.001$ \\
\hline Adjumani & & 0.9 & $0.7,1.0$ & 1.0 & $0.9,1.1$ & 0.7 & $0.6,0.8$ & \\
\hline Ikafe & & 0.8 & $0.6,0.9$ & 1.0 & $0.7,1.3$ & 0.6 & $0.5,0.7$ & \\
\hline Imvepi & & 0.8 & $0.4,1.1$ & 0.8 & $0.5,1.0$ & 0.8 & $0.4,1.2$ & \\
\hline Kiryandongo & & 1.5 & $1.0,2.0$ & 1.7 & $1.2,2.3$ & 1.3 & $0.8,1.7$ & \\
\hline Kyaka II & & 1.1 & $0.9,1.3$ & 1.2 & $1 \cdot 0,1 \cdot 4$ & 1.0 & $0.8,1.2$ & \\
\hline Kyangwali & & 1.3 & $1.2,1.5$ & 1.5 & $1.3,1.7$ & 1.1 & $1.0,1.2$ & \\
\hline Madi Okollo & & 0.8 & $0.7,1.0$ & 0.9 & $0.7,1.1$ & 0.7 & $0.6,0.9$ & \\
\hline Nakivale & & 1.2 & $0.9,1.5$ & 1.3 & $1.0,1.6$ & 1.2 & $0.9,1.6$ & \\
\hline Oruchinga & & 2.1 & $1.3,3.0$ & 2.5 & $1.6,3.5$ & 1.8 & $1.0,2.5$ & \\
\hline Palorinya & & 1.5 & $1.1,1.9$ & 1.8 & $1.5,2.1$ & 1.2 & $0.7,1.6$ & \\
\hline Rhino & & 0.8 & $0.3,1.3$ & 1.0 & $0.4,1.6$ & 0.7 & $0.3,1.1$ & \\
\hline Yemen & Kharaz & 2.1 & $1.3,2.8$ & 2.1 & $1.3,3.0$ & 2.0 & $1.4,2.7$ & \\
\hline Zambia & & 1.6 & $1.2,2.1$ & 1.8 & $1.3,2.2$ & 1.5 & $1.1,1.9$ & $<0.001$ \\
\hline Kala & & 1.0 & $0.8,1.2$ & 0.9 & $0.8,1.2$ & 1.0 & $0.8,1.2$ & \\
\hline Maheba & & 2.1 & $1.0,3.2$ & 2.3 & $1.1,3.6$ & 1.8 & $0.9,2.8$ & \\
\hline Mayukwayukwa & & 1.2 & $1.0,1.3$ & 1.4 & $1.2,1.6$ & 1.0 & $0.8,1.1$ & \\
\hline Mwange & & & 2.3 & $1.9,2.7$ & & 2.2 & $1.8,2.5$ & \\
\hline Asia & & 3.5 & $3.3,3.7$ & 3.8 & $3.6,4.0$ & 3.2 & $3.0,3.4$ & $<0.001$ \\
\hline Bangladesh & & 4.1 & $3.2,4.9$ & 4.2 & $3.2,5.2$ & 3.9 & $3.1,4.7$ & $<0.05$ \\
\hline Kutupalong & & 5.0 & $4.2,5.7$ & 5.1 & $4.2,6.1$ & 4.7 & $4.1,5.4$ & \\
\hline Nayapara & & 3.2 & $2.9,3.4$ & 3.3 & $3.1,3.6$ & 3.0 & $2.8,3.3$ & \\
\hline Nepal & & 3.5 & $3.3,3.8$ & 3.9 & $3.6,4.1$ & 3.2 & $2.9,3.4$ & $<0.001$ \\
\hline Beldangi I & & 3.0 & $2.5,3.4$ & 3.2 & $2.8,3.7$ & 2.7 & $2.3,3.1$ & \\
\hline Beldangi II & & 3.1 & $2.5,3.6$ & 3.4 & $2.8,4.0$ & 2.8 & $2.2,3.3$ & \\
\hline Beldangi II ext & & 3.4 & $2.8,3.9$ & 3.7 & $3.1,4.3$ & 3.0 & $2.5,3.5$ & \\
\hline Goldhap & & 4.4 & $3.7,5.2$ & 4.9 & $4.1,5.7$ & 4.0 & $3.3,4.7$ & \\
\hline Khudunabari & & 3.5 & $3.0,3.9$ & 3.8 & $3.4,4.2$ & 3.1 & $2.7,3.6$ & \\
\hline Sanishare & & 3.4 & $3.0,3.8$ & 3.7 & $3.3,4.1$ & 3.1 & $2.7,3.5$ & \\
\hline Timai & & 4.0 & $3.4,4.5$ & 4.3 & $3.7,4.9$ & 3.6 & $3.1,4.2$ & \\
\hline Thailand & & 3.4 & $3.1,3.7$ & 3.7 & $3.3,4.0$ & 3.1 & $2.9,3.4$ & $<0.001$ \\
\hline Ban Don Yang & & 3.8 & $3.5,4.1$ & 4.1 & $3.7,4.4$ & 3.6 & $3.3,3.8$ & \\
\hline Ban Mae Surin & & 5.3 & $4.5,6.0$ & 5.8 & $5.0,6.6$ & 4.8 & $4.1,5.5$ & \\
\hline Ban Mai Nai Soi & & 3.2 & $2.9,3.5$ & 3.3 & $3.0,3.5$ & 3.1 & $2.8,3.4$ & \\
\hline Mae La & & 2.4 & $2.1,2.7$ & 2.4 & $2.2,2.7$ & 2.3 & $2.0,2.7$ & \\
\hline Mae La Oon & & 3.5 & $3.3,3.8$ & 3.6 & $3.3,4.0$ & 3.4 & $3.1,3.8$ & \\
\hline Mae Ra Ma Luang & & 3.9 & $3.6,4.1$ & 4.2 & $4.0,4.5$ & 3.5 & $3.3,3.7$ & \\
\hline Nu Poh & & 2.5 & $2.4,2.6$ & 2.8 & $2.6,2.9$ & 2.2 & $2.1,2.3$ & \\
\hline Tham Hin & & 3.5 & $3.2,3.9$ & 3.9 & $3.5,4.3$ & 3.2 & $2.9,3.5$ & \\
\hline Umpiem Mai & & 2.5 & $2.3,2.6$ & 2.8 & $2.6,3.0$ & 2.1 & $2.0,2.3$ & \\
\hline All Regions & & 2.2 & $2.0,2.4$ & 2.4 & $2.3,2.6$ & 2.1 & $1.9,2.2$ & $<0.001$ \\
\hline \multirow{2}{*}{\multicolumn{2}{|c|}{ Asia - Africa Differential }} & 1.7 & $1.4,2.0$ & 1.8 & $1.6,2.1$ & 1.6 & $1.3,1.8$ & \\
\hline & & \multicolumn{2}{|c|}{$(p<0.001)$} & \multicolumn{2}{|c|}{$(p<0.001)$} & \multicolumn{2}{|c|}{$(p<0.001)$} & \\
\hline
\end{tabular}

* Values, Confidence Intervals and Significance are based on Generalized Estimating Equations, population-averaged model (Std. Err. adjusted for clustering on (amp) 
Table 3 Mean Proportion of New Outpatient Department Visits per Month by Refugees vs. Host Nationals, 2008-2009

\begin{tabular}{|c|c|c|c|c|c|c|}
\hline \multirow{2}{*}{ Region/Country/Camp } & & \multicolumn{2}{|c|}{ Refugee } & \multicolumn{2}{|c|}{ Host National } & \multirow[t]{2}{*}{ Ref - Host Difference $p$ Value } \\
\hline & & Pct * & $95 \% \mathrm{Cl}^{*}$ & Pct * & $95 \% \mathrm{Cl}^{*}$ & \\
\hline Africa & & 78.9 & $73.7,84.2$ & 21.1 & $15.8,26.3$ & $<.001$ \\
\hline Burundi & & 90.8 & $87.4,94.2$ & 9.2 & $5.8,12.6$ & $<.001$ \\
\hline Bwagiriza & & 88.9 & $78.6,99.1$ & 11.1 & $0.9,21.4$ & \\
\hline Gasorwe & & 91.5 & $85.2,97.9$ & 8.5 & $2.1,14.8$ & \\
\hline Gihinga & & 93.6 & $85.1,102.1$ & 6.4 & $-2.1,14.9$ & \\
\hline Musasa & & 89.3 & $83.9,94.6$ & 10.7 & $5.4,16.1$ & \\
\hline Cameroon & Langui & 96.7 & $91.1,102.2$ & 3.3 & $-2.2,8.9$ & $<.001$ \\
\hline Chad & & 88.1 & $85.9,90.2$ & 11.9 & $9.8,14.1$ & $<.001$ \\
\hline Amboko & & 98.9 & $98.1,99.7$ & 1.1 & $0.3,1.9$ & \\
\hline Amnabak & & 85.0 & $81.7,88.4$ & 15.0 & $11.6,18.3$ & \\
\hline Bredjing & & 95.3 & $93.5,97.1$ & 4.7 & $2.9,6.5$ & \\
\hline Djabal & & 94.5 & $90.8,98.1$ & 5.5 & $1.9,9.2$ & \\
\hline Dosseye & & 88.3 & $86.5,90.1$ & 11.7 & $9.9,13.5$ & \\
\hline Farchana & & 70.2 & $63.1,77.3$ & 29.8 & $22.7,36.9$ & \\
\hline Gaga & & 87.1 & $86.4,87.9$ & 12.9 & $12.1,13.6$ & \\
\hline Gondje & & 99.1 & $98.1,100.2$ & 0.9 & $-0.2,1.9$ & \\
\hline Goz Amer & & 90.3 & $88.3,92.2$ & 9.7 & $7.8,11.7$ & \\
\hline Kounoungou & & 84.3 & $82.5,86.1$ & 15.7 & $13.9,17.5$ & \\
\hline Mile & & 84.1 & $81.5,86.7$ & 15.9 & $13.3,18.5$ & \\
\hline Moula & & 93.3 & $91.3,95.3$ & 6.7 & $4.7,8.7$ & \\
\hline Oure Cassoni & & 84.4 & $81.9,86.9$ & 15.6 & $13.0,18.1$ & \\
\hline Treguine & & 84.0 & $80.5,87.5$ & 16.0 & $12.5,19.5$ & \\
\hline Yaroungou & & 81.6 & $78.5,84.8$ & 18.4 & $15.2,21.5$ & \\
\hline Djibouti & Ali Adde & 98.8 & $98.2,99.3$ & 1.2 & $0.7,1.8$ & $<.001$ \\
\hline Ethiopia & & 85.4 & $73.7,97.1$ & 14.6 & $2.9,26.3$ & $<.001$ \\
\hline Awbarre & & 98.3 & $97.6,99.0$ & 1.7 & $1.0,2.4$ & \\
\hline Fugnido & & 93.8 & $92.9,94.7$ & 6.2 & $5.3,7.1$ & \\
\hline Kebribeyah & & 91.4 & $90.1,92.7$ & 8.6 & $7.3,9.9$ & \\
\hline Sherkole & & 63.3 & $52.9,73.7$ & 36.7 & $26.3,47.1$ & \\
\hline Shimelba & & 72.0 & $67.8,76.1$ & 28.0 & $23.9,32.2$ & \\
\hline Guinea & Kouankan II & 94.8 & $91.2,98.5$ & 5.2 & $1.5,8.8$ & $<.001$ \\
\hline Kenya & & 97.2 & $91.9,102.5$ & 2.8 & $-2.5,8.1$ & $<.001$ \\
\hline Dagahaley & & 99.7 & $99.3,100.1$ & 0.3 & $-0.1,0.7$ & \\
\hline Hagadera & & 99.9 & $99.9,100.0$ & 0.1 & $0.0,0.1$ & \\
\hline Ifo & & 99.9 & $99.9,100.0$ & 0.0 & $0.0,0.1$ & \\
\hline Kakuma & & 87.2 & $86.1,88.4$ & 12.8 & $11.6,13.9$ & \\
\hline Rwanda & & 99.99 & $99.96,100$ & 0.01 & $0.0, .03$ & $<.001$ \\
\hline Gihembe & & 99.98 & $99.9,100$ & 0.0 & $0.0,0.1$ & \\
\hline Kiziba & & 91.1 & $88.7,93.6$ & 8.9 & $6.4,11.3$ & \\
\hline Nyabiheke & & 100 & & 0 & & \\
\hline Sudan & & 64.3 & $53.5,75.0$ & 35.7 & $25.0,44.5$ & $<.01$ \\
\hline Abuda & & 56.8 & $51.8,61.8$ & 43.2 & $38.2,48.2$ & \\
\hline Fau 5 & & 38.4 & $35.1,41.8$ & 61.6 & $58.2,64.9$ & \\
\hline Girba & & 57.8 & $56.1,59.4$ & 42.2 & $40.6,43.9$ & \\
\hline Kilo 26 & & 66.5 & $62.0,70.9$ & 33.5 & $29.1,38.0$ & \\
\hline Shagarab | || ||| & & 94.1 & $91.5,96.6$ & 5.9 & $3.3,8.5$ & \\
\hline Suki & & 36.7 & $35.4,38.0$ & 63.3 & $62.0,64.6$ & \\
\hline Um Gargour & & 82.9 & $68.6,97.1$ & 17.1 & $2.9,31.4$ & \\
\hline Wad Sharifey & & 68.6 & $65.4,71.8$ & 31.4 & $28.2,34.6$ & \\
\hline Tanzania & & 93.3 & $91.6,95.0$ & 6.7 & $5.0,8.4$ & $<.001$ \\
\hline
\end{tabular}


Table 3 Mean Proportion of New Outpatient Department Visits per Month by Refugees vs. Host Nationals, 2008-2009 (Continued)

\begin{tabular}{|c|c|c|c|c|c|c|}
\hline Lugufu & & 95.0 & $94.0,96.1$ & 5.0 & $3.9,6.0$ & \\
\hline Lukole & & 82.9 & $79.1,86.6$ & 17.1 & $13.4,20.9$ & \\
\hline Mtabila & & 94.9 & $94.2,95.6$ & 5.1 & $4.4,5.8$ & \\
\hline Nduta & & 95.7 & $94.1,97.2$ & 4.3 & $2.8,5.9$ & \\
\hline Nyarugusu & & 92.7 & $91.7,93.6$ & 7.3 & $6.4,8.3$ & \\
\hline Uganda & & 44.1 & $33.8,54.4$ & 55.9 & $45.6,66.2$ & $<.26$ \\
\hline Adjumani & & 29.8 & $16.1,43.4$ & 70.2 & $56.6,83.9$ & \\
\hline Ikafe & & 12.7 & $-3.8,29.1$ & 87.4 & $70.9,103.8$ & \\
\hline Imvepi & & 30.7 & $19.0,42.4$ & 69.3 & $57.6,81.0$ & \\
\hline Kiryandongo & & 56.9 & $53.2,60.6$ & 43.1 & $39.4,46.8$ & \\
\hline Kyaka II & & 63.5 & $60.0,67.1$ & 36.5 & $32.9,40.0$ & \\
\hline Kyangwali & & 54.0 & $48.7,59.3$ & 46.0 & $40.7,51.3$ & \\
\hline Madi Okollo & & 41.6 & $2.7,80.4$ & 58.4 & $19.6,97.3$ & \\
\hline Nakivale & & 89.7 & $85.8,93.7$ & 10.3 & $6.3,14.2$ & \\
\hline Oruchinga & & 27.6 & $19.5,35.7$ & 72.4 & $64.3,80.5$ & \\
\hline Palorinya & & 33.8 & $15.3,52.4$ & 66.2 & $47.6,84.7$ & \\
\hline Rhino & & 20.8 & $12.1,29.4$ & 79.2 & $70.6,87.9$ & \\
\hline Yemen & Kharaz & 69.7 & $65.9,73.5$ & 30.3 & $26.5,34.1$ & $<.001$ \\
\hline Zambia & & 88.5 & $82.5,94.5$ & 11.5 & $5.5,17.5$ & $<.001$ \\
\hline Kala & & 92.0 & $90.4,93.6$ & 8.0 & $6.4,9.6$ & \\
\hline Maheba & & 76.1 & $71.3,80.9$ & 23.9 & $19.1,28.7$ & \\
\hline Mayukwayukwa & & 85.5 & $80.5,90.6$ & 14.5 & $9.4,19.5$ & \\
\hline Mwange & & 98.5 & $98.0,99.1$ & 1.5 & $0.9,2.0$ & \\
\hline Asia & & 97.6 & $96.8,98.4$ & 2.4 & $1.6,3.2$ & $<.001$ \\
\hline Bangladesh & & 97.4 & $96.0,98.9$ & 2.6 & $1.1,4.0$ & $<.001$ \\
\hline Kutupalong & & 98.2 & $95.3,101.1$ & 1.8 & $-1.1,4.7$ & \\
\hline Nayapara & & 96.8 & $96.0,97.6$ & 3.2 & $2.4,4.0$ & \\
\hline Nepal & & 97.8 & $96.4,99.2$ & 2.2 & $0.8,3.6$ & $<.001$ \\
\hline Beldangi I & & 99.4 & $99.1,99.7$ & 0.6 & $0.3,0.9$ & \\
\hline Beldangi II & & 99.9 & $99.95,100$ & 0.0 & $0.0,0.0$ & \\
\hline Beldangi II ext & & 99.8 & $99.7,99.9$ & 0.2 & $0.1,0.3$ & \\
\hline Goldhap & & 97.8 & $97.5,98.1$ & 2.2 & $1.9,2.5$ & \\
\hline Khudunabari & & 94.5 & $93.6,95.3$ & 5.5 & $4.7,6.4$ & \\
\hline Sanishare & & 99.9 & $99.8,99.9$ & 0.1 & $0.1,0.2$ & \\
\hline Timai & & 93.9 & $93.1,94.7$ & 6.1 & $5.3,6.9$ & \\
\hline Thailand & & 97.5 & $96.3,98.6$ & 2.5 & $1.4,3.7$ & $<.001$ \\
\hline Ban Don Yang & & 96.9 & $95.6,98.1$ & 3.1 & $1.9,4.4$ & \\
\hline Ban Mae Surin & & 99.9 & $99.9,99.9$ & 0.0 & $0.0,0.1$ & \\
\hline Ban Mai Nai Soi & & 99.9 & $99.9,100.0$ & 0.0 & $0.0,0.0$ & \\
\hline Mae La & & 96.4 & $95.9,97.0$ & 3.6 & $3.0,4.1$ & \\
\hline Mae La Oon & & 97.3 & $96.9,97.8$ & 2.7 & $2.2,3.1$ & \\
\hline Mae Ra Ma Luang & & 98.3 & $98.0,98.5$ & 1.7 & $1.5,2.0$ & \\
\hline Nu Poh & & 90.2 & $89.1,91.3$ & 9.8 & $8.8,10.9$ & \\
\hline Tham Hin & & 99.9 & $99.8,99.9$ & 0.1 & $0.1,0.2$ & \\
\hline Umpiem Mai & & 99.3 & $99.2,99.5$ & 0.7 & $0.5,0.8$ & \\
\hline \multirow{3}{*}{\multicolumn{2}{|c|}{$\begin{array}{l}\text { All Regions } \\
\text { Asia - Africa Differential ( } p \text {-value) }\end{array}$}} & 82.9 & $78.5,87.3$ & 17.1 & $12.7,21.5$ & $<.001$ \\
\hline & & 18.6 & $9.2,28.0$ & & & \\
\hline & & $(p<.001)$ & & & & \\
\hline
\end{tabular}

* Values, Confidence Intervals and Significance are based on Generalized Estimating Equations, population-averaged model (Std. Err. adjusted for clustering on Camp) 
Table 4 Percent of New Outpatient Department Visits by Females, Refugee vs Host Country Patients, 2008-2009

\begin{tabular}{|c|c|c|c|c|c|c|c|c|c|c|}
\hline \multirow[b]{2}{*}{ Region/Country/Camp } & \multicolumn{3}{|c|}{ All } & \multicolumn{3}{|c|}{ Refugee } & \multicolumn{3}{|c|}{ Host } & \multirow{2}{*}{$\begin{array}{l}\text { Pct OPD } \\
\text { Female } \\
\text { Ref - Host } \\
\text { Difference } \\
\text { p Value* }\end{array}$} \\
\hline & & $\begin{array}{l}\text { Percent } \\
\text { OPD Visits } \\
\text { Female* }\end{array}$ & $95 \% \mathrm{Cl}^{*}$ & $\begin{array}{c}\text { Pct. } \\
\text { Refugee } \\
\text { Pop. } \\
\text { Female * }\end{array}$ & $\begin{array}{c}\text { Pct. OPD } \\
\text { Visits } \\
\text { Female* }\end{array}$ & $95 \% \mathrm{Cl}^{*}$ & $\begin{array}{c}\text { National } \\
\text { Pct Pop } \\
\text { Female } \\
* *\end{array}$ & $\begin{array}{l}\text { Pct. OPD } \\
\text { Visits } \\
\text { Female* }\end{array}$ & $95 \% \mathrm{Cl}^{*}$ & \\
\hline Africa & & 54.4 & $53.9,54.9$ & 51.1 & 54.8 & $54.4,55.3$ & 50 & 51.7 & $50.5,52.8$ & $p<.001$ \\
\hline Burundi & & 54.2 & $53.0,55.4$ & 51.2 & 53.9 & $52.9,55.0$ & 51 & 53.0 & $48.9,57.2$ & \\
\hline Bwagiriza & & 53.1 & $51.7,54.5$ & 51.1 & 53.2 & $51.9,54.5$ & & 42.4 & $26.0,58.9$ & \\
\hline Gasorwe & & 54.9 & $53.6,56.2$ & 52.2 & 54.3 & $53.5,55.1$ & & 54.1 & $46.3,62.0$ & \\
\hline Gihinga & & 56.2 & $54.8,57.5$ & 50.6 & 56.0 & $54.8,57.2$ & & 56.2 & $50.8,61.6$ & \\
\hline Musasa & & 52.2 & $49.9,54.4$ & 50.5 & 51.8 & $50.0,53.6$ & & 53.9 & $49.6,58.3$ & \\
\hline Cameroon & Langui & 51.6 & $49.6,53.6$ & 48.8 & 51.7 & $49.6,53.9$ & 50 & 45.5 & $32.9,58.2$ & \\
\hline Chad & & 53.9 & $53.2,54.6$ & 54.9 & 54.4 & $53.7,55.1$ & 50 & 48.4 & $46.1,50.7$ & $\mathrm{p}<.001$ \\
\hline Amboko & & 54.6 & $51.4,57.8$ & 53.5 & 54.9 & $51.6,58.1$ & & 35.5 & $26.5,44.5$ & $p<.001$ \\
\hline Amnabak & & 55.1 & $54.0,56.2$ & 61.3 & 55.1 & $53.9,56.3$ & & 54.8 & $52.3,57.2$ & \\
\hline Bredjing & & 51.1 & $48.8,53.5$ & 54.2 & 52.5 & $51.4,53.6$ & & 34.3 & $2,8,65.9$ & \\
\hline Djabal & & 52.6 & $51.5,53.7$ & 54.4 & 53.0 & $51.7,54.2$ & & 45.7 & $42.0,49.4$ & $p<.001$ \\
\hline Dosseye & & 57.7 & $56.8,58.6$ & 54.8 & 59.2 & $58.6,59.7$ & & 46.3 & $42.1,50.6$ & $p<.001$ \\
\hline Farchana & & 48.0 & $45.1,50.8$ & 55.3 & 49.2 & $45.9,52.4$ & & 45.8 & $43.7,47.9$ & $p<.05$ \\
\hline Gaga & & 52.7 & $51.6,53.9$ & 54.4 & 53.0 & $51.9,54.1$ & & 51.3 & $49.3,53.4$ & \\
\hline Gondje & & 53.2 & $51.0,55.3$ & 51.6 & 53.3 & $51.2,55.4$ & & 39.5 & $26.7,52.3$ & $p<.05$ \\
\hline Goz Amer & & 52.7 & $51.3,54.1$ & 53.3 & 53.0 & $51.6,54.3$ & & 49.3 & $47.8,50.7$ & $p<.001$ \\
\hline Kounoungou & & 55.4 & $54.4,56.3$ & 56.8 & 55.1 & $54.3,55.9$ & & 56.6 & $54.4,58.8$ & \\
\hline Mile & & 56.7 & $55.3,58.2$ & 56.2 & 57.4 & $56.1,58.7$ & & 52.8 & $50.7,54.9$ & $p<.001$ \\
\hline Moula & & 53.0 & $51.3,54.7$ & 49.5 & 53.4 & $51.3,55.5$ & & 44.8 & $37.3,52.3$ & \\
\hline Oure Cassoni & & 57.7 & $55.3,60.1$ & 60.2 & 58.4 & $55.1,61.6$ & & 54.3 & $51.9,56.7$ & \\
\hline Treguine & & 49.3 & $48.4,50.1$ & 51.3 & 49.5 & $48.4,50.5$ & & 48.4 & $46.6,50.2$ & \\
\hline Yaroungou & & 54.7 & $52.6,56.9$ & 53.2 & 56.0 & $52.3,59.7$ & & 48.1 & $41.2,55.0$ & \\
\hline Djibouti & Ali Adde & 56.2 & $55.2,57.2$ & 50.8 & 56.3 & $55.2,57.4$ & 50 & 46.4 & $39.6,53.2$ & $\mathrm{p}<.01$ \\
\hline Ethiopia & & 52.6 & $49.5,55.7$ & 46.2 & 52.3 & $48.7,56.0$ & 50 & 50.3 & $48.6,51.9$ & \\
\hline Awbarre & & 57.7 & $56.0,59.4$ & 50.9 & 57.7 & $56.1,59.4$ & & 52.9 & $47.8,58.0$ & \\
\hline Fugnido & & 56.3 & $55.0,57.7$ & 54.9 & 56.8 & $55.4,58.3$ & & 49.3 & $46.4,52.2$ & $p<.001$ \\
\hline Kebribeyah & & 54.8 & $53.8,55.8$ & 50.4 & 55.0 & $54.0,55.9$ & & 53.0 & $50.2,55.8$ & \\
\hline Sherkole & & 49.2 & $45.1,53.2$ & 45.2 & 48.4 & $42.9,54.0$ & & 49.3 & $47.8,50.9$ & \\
\hline Shimelba & & 42.4 & $41.7,43.1$ & 28.3 & 40.8 & $40.2,41.4$ & & 46.9 & $43.9,50.0$ & $p<.001$ \\
\hline Guinea & $\begin{array}{l}\text { Kouankan } \\
\text { ॥ }\end{array}$ & 56.5 & $54.2,58.7$ & 53.2 & 56.7 & $54.2,59.1$ & 50 & 54.9 & $51.6,58.2$ & \\
\hline Kenya & & 50.3 & $49.3,51.2$ & 47.8 & 50.3 & $49.3,51.3$ & 50 & 49.2 & $40.5,58.0$ & \\
\hline Dagahaley & & 51.3 & $50.5,52.2$ & 49.4 & 51.3 & $50.5,52.2$ & & 63.3 & $33.7,93.0$ & \\
\hline Hagadera & & 51.7 & $50.6,52.7$ & 48.7 & 51.7 & $50.7,52.7$ & & 47.5 & $32.9,62.1$ & \\
\hline Ifo & & 50.9 & $48.6,53.2$ & 48.9 & 50.9 & $48.6,53.2$ & & 42.7 & $18.2,67.2$ & \\
\hline Kakuma & & 47.1 & $46.6,47.7$ & 44.1 & 47.3 & $46.5,48.1$ & & 46.6 & $44.7,48.5$ & \\
\hline Rwanda & & 56.4 & $55.0,57.9$ & 55.2 & 56.4 & $55.0,57.9$ & 52 & - & - & \\
\hline Gihembe & & 56.2 & $54.8,57.6$ & 54.9 & 56.2 & $54.8,57.6$ & & - & - & \\
\hline Kiziba & & 58.3 & $56.3,60.3$ & 55.0 & 58.3 & $56.4,60.2$ & & 57.9 & $54.2,61.6$ & \\
\hline Nyabiheke & & 54.3 & $51.9,56.8$ & 55.9 & 54.3 & $51.9,56.8$ & & - & - & \\
\hline Sudan & & 55.6 & $54.0,57.3$ & 50.0 & 57.3 & $56.6,58.0$ & 50 & 52.4 & $46.7,58.0$ & \\
\hline Abuda & & 58.3 & $55.1,61.5$ & 48.7 & 60.5 & $59.0,62.0$ & & 55.7 & $50.6,60.7$ & $p<.05$ \\
\hline Fau 5 & & 52.8 & $51.3,54.3$ & 54.6 & 56.5 & $54.9,58.0$ & & 50.8 & $48.7,52.9$ & $p<.001$ \\
\hline Girba & & 54.6 & $46.3,62.9$ & 50.2 & 58.0 & $57.2,58.8$ & & 49.5 & $29.6,69.4$ & \\
\hline
\end{tabular}


Table 4 Percent of New Outpatient Department Visits by Females, Refugee vs Host Country Patients, 2008-2009 (Continued)

\begin{tabular}{|c|c|c|c|c|c|c|c|c|c|}
\hline Kilo 26 & 53.7 & $47.4,59.9$ & 45.2 & 55.2 & $54.6,55.8$ & & 49.5 & $30.5,68.5$ & \\
\hline Shagarab | || ||| & 57.6 & $53.1,62.2$ & 49.5 & 58.2 & $54.8,61.6$ & & 56.0 & $30.6,81.5$ & \\
\hline Suki & 56.3 & $54.2,58.4$ & 48.5 & 55.7 & $53.8,57.7$ & & 56.6 & $53.2,60.1$ & \\
\hline Um Gargour & 54.8 & $51.6,58.0$ & 47.7 & 56.6 & $55.7,57.5$ & & 46.8 & $28.9,64.8$ & \\
\hline Wad Sharifey & 56.8 & $56.0,57.5$ & 55.9 & 57.6 & $57.0,58.2$ & & 55.2 & $53.0,57.4$ & $p<.05$ \\
\hline Tanzania & 52.8 & $51.7,53.9$ & 50.7 & 52.9 & $51.8,54.0$ & 50 & 51.2 & $49.0,53.3$ & $p<.05$ \\
\hline Lugufu & 49.4 & $47.5,51.2$ & 51.0 & 49.3 & $47.4,51.2$ & & 48.9 & $43.5,54.3$ & \\
\hline Lukole & 55.0 & $54.2,55.7$ & 49.4 & 55.8 & $54.9,56.7$ & & 51.0 & $50.9,51.1$ & $p<.001$ \\
\hline Mtabila & 55.3 & $54.8,55.8$ & 50.5 & 55.3 & $54.8,55.8$ & & 55.5 & $53.0,58.0$ & \\
\hline Nduta & 54.8 & $53.6,56.0$ & 50.7 & 54.8 & $53.5,56.1$ & & 53.4 & $50.3,56.5$ & \\
\hline Nyarugusu & 51.5 & $50.5,52.5$ & 51.1 & 51.8 & $50.8,52.8$ & & 47.2 & $44.9,49.5$ & $p<.001$ \\
\hline Uganda & 57.1 & $56.2,58.0$ & 50.2 & 57.5 & $56.5,58.5$ & 50 & 56.6 & $55.4,57.7$ & \\
\hline Adjumani & 57.0 & $55.3,58.7$ & 51.3 & 58.8 & $57.2,60.6$ & & 55.8 & $53.3,58.3$ & \\
\hline Ikafe & 55.3 & $53.2,57.4$ & 46.0 & 58.3 & $53.7,62.9$ & & 53.6 & $49.8,57.3$ & \\
\hline Imvepi & 54.4 & $50.1,58.7$ & 51.2 & 55.7 & $48.7,62.6$ & & 55.5 & $52.0,59.1$ & \\
\hline Kiryandongo & 56.7 & $54.8,58.7$ & 49.8 & 57.6 & $55.6,59.6$ & & 56.1 & $53.3,59.0$ & \\
\hline Kyaka II & 56.2 & $54.1,58.2$ & 50.5 & 54.5 & $53.2,55.9$ & & 58.0 & $54.3,61.7$ & \\
\hline Kyangwali & 56.6 & $54.9,58.3$ & 50.3 & 58.0 & $56.8,59.3$ & & 55.1 & $52.6,57.7$ & $p<.001$ \\
\hline Madi Okollo & 60.9 & $56.3,65.6$ & 49.6 & 55.1 & $50.8,59.4$ & & 59.6 & $52.6,66.7$ & \\
\hline Nakivale & 56.4 & $54.2,58.7$ & 51.1 & 56.2 & $53.8,58.7$ & & 56.5 & $53.8,59.3$ & \\
\hline Oruchinga & 57.7 & $54.5,60.9$ & 49.7 & 57.7 & $56.7,58.7$ & & 57.0 & $52.5,61.6$ & \\
\hline Palorinya & 59.8 & $56.7,62.9$ & 51.8 & 61.9 & $59.6,64.2$ & & 58.2 & $54.2,62.1$ & $p<.01$ \\
\hline Rhino & 57.1 & $52.7,61.5$ & 48.0 & 57.4 & $54.1,60.8$ & & 56.3 & $50.4,62.1$ & \\
\hline Yemen & 53.6 & $51.5,55.7$ & 50.9 & 53.3 & $51.2,55.4$ & 49 & 53.8 & $52.0,55.7$ & \\
\hline Zambia & 53.9 & $52.5,55.3$ & 49.9 & 54.3 & $52.9,55.7$ & 50 & 51.4 & $49.2,53.6$ & $p<.01$ \\
\hline Kala & 50.9 & $49.6,52.2$ & 50.6 & 51.4 & $50.1,52.7$ & & 47.6 & $43.4,51.7$ & \\
\hline Maheba & 52.6 & $50.4,54.8$ & 48.8 & 53.1 & $50.7,55.6$ & & 49.2 & $45.5,52.9$ & $p<.05$ \\
\hline Mayukwayukwa & 57.6 & $55.5,59.7$ & 49.7 & 58.2 & $55.8,60.5$ & & 55.1 & $53.8,56.5$ & $p<.01$ \\
\hline Mwange & 54.4 & $53.4,55.4$ & 50.6 & 54.4 & $53.3,55.4$ & & 53.5 & $48.3,58.6$ & \\
\hline Asia & 53.3 & $52.9,53.8$ & 49.4 & 53.4 & $52.9,53.9$ & 50 & 48.5 & $46.4,50.5$ & $\mathrm{p}<.001$ \\
\hline Bangladesh & 53.3 & $51.9,54.6$ & 51.5 & 53.7 & $52.2,55.1$ & 49 & 37.4 & $32.5,42.3$ & $p<.001$ \\
\hline Kutupalong & 52.8 & $51.0,54.7$ & 51.2 & 53.2 & $51.1,55.2$ & & 37.1 & $28.5,45.7$ & $p<.001$ \\
\hline Nayapara & 53.7 & $51.8,55.6$ & 51.9 & 54.2 & $52.4,56.1$ & & 37.6 & $32.9,42.4$ & $p<.001$ \\
\hline Nepal & 54.1 & $53.7,54.5$ & 49.2 & 54.2 & $53.8,54.5$ & 50 & 50.2 & $47.0,53.4$ & $p<.05$ \\
\hline Beldangi I & 54.0 & $53.2,54.8$ & 49.2 & 54.0 & $53.2,54.8$ & & 51.8 & $44.4,59.2$ & \\
\hline Beldangi II & 54.4 & $52.9,56.0$ & 49.2 & 54.4 & $52.9,56.0$ & & 59.1 & $47.3,70.8$ & \\
\hline Beldangi II ext & 54.4 & $53.5,55.4$ & 49.0 & 54.4 & $53.5,55.4$ & & 51.3 & $39.9,62.7$ & \\
\hline Goldhap & 53.9 & $53.0,54.8$ & 48.8 & 53.9 & $53.0,54.9$ & & 51.8 & $49.9,53.8$ & \\
\hline Khudunabari & 54.5 & $53.5,55.5$ & 49.8 & 54.6 & $53.6,55.7$ & & 52.2 & $51.0,53.4$ & $p<.01$ \\
\hline Sanishare & 54.2 & $53.6,54.9$ & 49.3 & 54.2 & $53.6,54.9$ & & 39.6 & $27.9,51.3$ & $p<.05$ \\
\hline Timai & 53.2 & $52.3,54.1$ & 49.0 & 53.3 & $52.3,54.2$ & & 51.9 & $50.8,52.9$ & $p<.05$ \\
\hline Thailand & 52.7 & $51.9,53.5$ & 49.1 & 52.7 & $52.0,53.5$ & 51 & 50.1 & $47.5,52.7$ & $p<.05$ \\
\hline Ban Don Yang & 54.3 & $53.3,55.2$ & 51.0 & 54.3 & $53.3,55.3$ & & 55.0 & $50.2,59.7$ & \\
\hline Ban Mae Surin & 53.3 & $52.2,54.4$ & 48.4 & 53.3 & $52.3,54.4$ & & 52.1 & $12.3,91.8$ & \\
\hline $\begin{array}{l}\text { Ban Mai Nai } \\
\text { Soi }\end{array}$ & 49.5 & $48.7,50.3$ & 48.2 & 49.5 & $48.7,50.3$ & & - & - & \\
\hline Mae La & 50.5 & $49.1,51.9$ & 49.3 & 50.4 & $49.1,51.8$ & & 50.8 & $48.4,53.2$ & \\
\hline Mae La Oon & 49.6 & $45.5,53.8$ & 49.1 & 49.7 & $45.5,53.9$ & & 45.5 & $41.8,49.3$ & $p<.01$ \\
\hline $\begin{array}{l}\text { Mae Ra Ma } \\
\text { Luang }\end{array}$ & 54.4 & $53.2,55.5$ & 49.9 & 54.5 & $53.3,55.6$ & & 49.2 & $47.1,51.3$ & $p<.001$ \\
\hline Nu Poh & 53.7 & $52.7,54.7$ & 48.2 & 53.8 & $52.8,54.8$ & & 52.8 & $51.7,53.9$ & \\
\hline Tham Hin & 53.9 & $52.9,54.9$ & 48.9 & 53.9 & $52.9,54.9$ & & 46.7 & $33.7,59.7$ & \\
\hline
\end{tabular}


Table 4 Percent of New Outpatient Department Visits by Females, Refugee vs Host Country Patients, 2008-2009 (Continued)

\begin{tabular}{|c|c|c|c|c|c|c|c|c|c|}
\hline Umpiem Mai & 55.3 & $54.4,56.2$ & 48.5 & 55.3 & $54.5,56.2$ & & 49.9 & $43.3,56.3$ & \\
\hline All Regions & 54.1 & $53.8,54.5$ & 50.7 & 54.5 & $54.1,54.9$ & 50 & 50.9 & $49.9,51.9$ & $p<.001$ \\
\hline $\begin{array}{l}\text { Asia - Africa } \\
\text { Differential (p-value) }\end{array}$ & $\begin{array}{c}-1.1 \\
(p<.05)\end{array}$ & $-2.0,-0.2$ & $\begin{array}{c}-1.7(p< \\
.10)\end{array}$ & -1.4 & $\begin{array}{l}-2.3,-0.6 \\
(p<.01)\end{array}$ & 0 & -3.2 & $\begin{array}{l}-5.5,-0.9 \\
(p<.01)\end{array}$ & \\
\hline
\end{tabular}

* Values, Confidence Intervals and Significance are based on Generalized Estimating Equations, population-averaged model (Std. Err. adjusted for clustering on Camp); only p-values significant to the .05 level or less are provided.

** Source: World Bank, Health, Nutrition and Population database estimates for 2008 http://databank.worldbank.org.

was higher in African settlements than in Asian settlements. This regional difference was greater among host nationals than among refugees.

\section{Proportion of New Outpatient Diagnoses per Month Proportion of new outpatient diagnoses by age}

Table 5 depicts the mean proportion of new outpatient diagnosis each month attributable to children under five years of age. Table 5 also compares this same proportion between refugees and host nationals utilizing settlement outpatient services. Because the UNHCR's Health Information System database does not document new visits by age group, we have included analysis of new outpatient diagnoses to allow us to look at age patterns in use of services. By looking at diagnoses, we understand that one person may have multiple diagnoses on a single visit; there is not a one to one ratio between visits and diagnoses. The database available only allows for age-specific analysis for two groups: (1) under five years; or, (2) five years of age or higher.

Across all settlements reporting to the UNHCR database, the percent of the refugee population that was less than five years of age is $16 \%$ on average (Table 5). The average under-five year population for Asia was significantly lower than the overall average at $12 \%$. In general, the Asian population living in refugee settlements was older than the population living African settlements. However, there was considerable variation among countries. For example, Bangladesh, Tanzania, Rwanda, Yemen and Zambia had an average under-five refugee population greater than 19\%, while Nepal and Sudan had rates as low as $8-9 \%$. National estimates of the size of the under-five population in host countries are also provided in Table 5 for comparison (this information is not available at the local level for host populations using refugee settlement health services). Across all countries contributing to the database, the estimated under-five population is an average of $14 \%$ (weighted for population size of included countries). For African countries, the average is $16 \%$; it is $10 \%$ for Asian countries. There is substantial variation between countries in the estimated proportion less than five years of age: from $7 \%$ in Thailand to over $19 \%$ in Uganda.
Proportion of new outpatient diagnoses attributable to children less than five years of age by status (refugee vs. host national)

Although under-fives make up $16 \%$ of refugee settlement populations on average, they represent $36 \%$ of all outpatient diagnoses among refugees. Very similar, although the national estimates of the size of the underfive population among host countries averages at $14 \%$, under-fives represent $36 \%$ of outpatient diagnoses among host nationals.

The proportion of outpatient diagnoses attributable to under-fives among host nationals was slightly higher (39\%), on average, than the proportion of outpatient diagnoses attributable to under-fives among refugees (37\%). This pattern was consistent across most African countries except for Burundi. In Asia, in constrast, the proportion of outpatient diagnoses attributable to under-fives among host nationals was much lower (24\%) than the proportion of outpatient diagnoses attributable to under-fives among refugees (30\%). Overall, the proportion of all new outpatient diagnoses attributable to under-fives was lower in Asia (30\%) as compared to Africa (39\%).

\section{Discussion}

Several studies have compared use of reproductive health and HIV health services by refugees versus host communities. However, there is limited information in the literature about general patterns of use of refugee health facilities by refugees and members of host communities. The availability of a database, that combines reports from the majority of refugee settlements supported by UNHCR and partners, provides a unique opportunity to explore how services differ between gender and age groups, and between refugees and host nationals who utilize the health services of the settlements. The structure of the database also allows us to look at overall patterns and to compare and contrast these patterns between and within regions and countries.

\section{Utilization rates}

Utilization rates among refugees vary between regions. In Africa, the average utilization rate is 1.8. However, in 
Table 5 Percent of Outpatient Department Diagnoses by Children Less than Five Years of Age (U5), Refugee vs Host Country Patients, 2008-2009

\begin{tabular}{|c|c|c|c|c|c|c|c|c|c|c|}
\hline \multirow[b]{2}{*}{ Region/Country/Camp } & \multicolumn{3}{|c|}{ All } & \multicolumn{3}{|c|}{ Refugee } & \multicolumn{3}{|c|}{ Host } & \multirow{2}{*}{$\begin{array}{c}\text { Pct OPD } \\
\text { U5 } \\
\text { Ref - Host } \\
\text { Difference } \\
\text { p Value* }\end{array}$} \\
\hline & & $\begin{array}{c}\text { Percent } \\
\text { OPD } \\
\text { Diagnoses } \\
\text { U5* }\end{array}$ & $95 \% \mathrm{Cl}^{*}$ & $\begin{array}{c}\text { Pct. } \\
\text { Refugee } \\
\text { Pop. } \\
\text { U5 * }\end{array}$ & $\begin{array}{c}\text { Pct. OPD } \\
\text { Diagnoses } \\
\text { U5* }\end{array}$ & $95 \% \mathrm{Cl}^{*}$ & $\begin{array}{l}\text { National } \\
\text { Pct. Pop. } \\
\text { U5 ** }\end{array}$ & $\begin{array}{l}\text { Pct. OPD } \\
\text { Diagnoses } \\
\text { U5* }\end{array}$ & $95 \% \mathrm{Cl}^{*}$ & \\
\hline Africa & & 38.6 & $37.6,39.5$ & 16.9 & 37.4 & $36.3,38.5$ & 16.2 & 39.4 & $38.2,40.6$ & $p<.001$ \\
\hline Burundi & & 39.8 & $37.3,42.4$ & 19.4 & 40.7 & $38.0,43.4$ & 14.3 & 28.2 & $24.3,32.2$ & $\mathrm{p}<.001$ \\
\hline Bwagiriza & & 38.3 & $29.5,47.0$ & 23.4 & 38.8 & $30.3,47.2$ & & 23.4 & $7.6,39.3$ & $p<.01$ \\
\hline Gasorwe & & 40.3 & $39.1,41.6$ & 22.8 & 41.9 & $40.5,43.2$ & & 23.5 & $17.7,29.3$ & $p<.001$ \\
\hline Gihinga & & 35.8 & $33.9,37.7$ & 14.9 & 36.5 & $36.5,38.5$ & & 27.5 & $22.9,32.1$ & $p<.01$ \\
\hline Musasa & & 41.9 & $35.0,48.7$ & 18.6 & 42.3 & $35.2,49.5$ & & 37.5 & $32.2,42.7$ & $p<.001$ \\
\hline Cameroon & Langui & 26.6 & $21.1,32.0$ & 18.5 & 26.5 & $21.1,31.9$ & 15.8 & 29.6 & $20.2,38.9$ & \\
\hline Chad & & 41.7 & $40.2,43.3$ & 18.4 & 41.9 & $40.3,43.6$ & 18.2 & 39.4 & $37.1,41.8$ & \\
\hline Amboko & & 41.6 & $31.2,51.9$ & 12.2 & 41.6 & $31.2,52.1$ & & 28.5 & $16.7,40.3$ & \\
\hline Amnabak & & 36.3 & $34.1,38.5$ & 23.3 & 36.0 & $33.4,38.6$ & & 40.6 & $37.2,44.0$ & \\
\hline Bredjing & & 41.4 & $37.9,44.9$ & 19.0 & 40.2 & $37.8,42.7$ & & 53.5 & $37.3,69.7$ & \\
\hline Djabal & & 39.6 & $36.7,42.5$ & 21.2 & 39.7 & $36.9,42.4$ & & 37.3 & $29.4,45.3$ & \\
\hline Dosseye & & 40.8 & $37.5,44.1$ & 19.7 & 38.4 & $34.8,42.1$ & & 53.5 & $45.1,61.9$ & $p<.01$ \\
\hline Farchana & & 44.1 & $39.8,48.3$ & 17.2 & 45.2 & $41.0,49.5$ & & 40.1 & $36.8,43.4$ & $p<.001$ \\
\hline Gaga & & 44.7 & $41.3,48.2$ & 20.9 & 45.8 & $42.0,49.6$ & & 37.7 & $30.7,44.8$ & $p<.05$ \\
\hline Gondje & & 43.2 & $30.9,55.4$ & 11.3 & 43.3 & $31.1,55.5$ & & 26.5 & $12.2,40.7$ & \\
\hline Goz Amer & & 43.7 & $40.1,47.3$ & 22.2 & 44.2 & $39.8,48.5$ & & 41.2 & $37.9,44.6$ & \\
\hline Kounoungou & & 40.2 & $38.7,41.8$ & 17.4 & 41.2 & $39.6,42.8$ & & 35.8 & $30.6,41.0$ & \\
\hline Mile & & 41.7 & $38.6,44.8$ & 17.4 & 43.1 & $39.0,47.2$ & & 35.0 & $30.2,39.8$ & \\
\hline Moula & & 37.5 & $23.7,51.2$ & 25.0 & 38.7 & $24.0,53.4$ & & 26.6 & $22.8,30.3$ & $p<.01$ \\
\hline Oure Cassoni & & 41.7 & $39.5,43.9$ & 15.6 & 42.0 & $39.4,44.5$ & & 41.4 & $35.7,47.1$ & \\
\hline Treguine & & 43.2 & $39.6,46.9$ & 19.1 & 44.3 & $40.1,48.6$ & & 38.7 & $33.3,44.1$ & \\
\hline Yaroungou & & 43.9 & $39.6,48.2$ & 18.0 & 42.2 & $36.4,48.1$ & & 49.6 & $44.4,54.9$ & $p<.05$ \\
\hline Djibouti & Ali Adde & 34.5 & $31.0,38.1$ & 16.1 & 34.5 & $31.0,38.1$ & 13.5 & 34.0 & $16.3,51.7$ & \\
\hline Ethiopia & & 41.8 & $39.9,43.7$ & 17.7 & 41.4 & $39.3,43.4$ & 16.5 & 40.6 & $35.1,46.0$ & \\
\hline Awbarre & & 47.7 & $44.7,50.7$ & 19.7 & 48.3 & $45.2,51.5$ & & 41.0 & $27.4,54.6$ & \\
\hline Fugnido & & 40.9 & $37.7,44.1$ & 23.8 & 42.1 & $38.6,45.6$ & & 27.2 & $16.4,38.0$ & $p<.05$ \\
\hline Kebribeyah & & 38.3 & $36.3,40.3$ & 20.6 & 37.8 & $35.7,39.8$ & & 43.1 & $38.1,48.1$ & $p<.05$ \\
\hline Sherkole & & 39.5 & $36.5,42.6$ & 18.1 & 38.1 & $35.5,40.7$ & & 42.2 & $36.2,48.7$ & \\
\hline Shimelba & & 43.2 & $40.0,46.3$ & 9.0 & 40.7 & $37.0,44.4$ & & 48.9 & $45.1,52.8$ & $p<.01$ \\
\hline Guinea & $\begin{array}{l}\text { Kouankan } \\
\text { ॥ }\end{array}$ & 28.5 & $26.5,30.6$ & 14.2 & 27.8 & $26.1,29.6$ & 16.7 & 35.7 & $21.4,49.9$ & \\
\hline Kenya & & 39.5 & $37.8,41.2$ & 15.4 & 39.3 & $37.4,41.1$ & 16.9 & 39.7 & $33.5,46.0$ & \\
\hline Dagahaley & & 40.6 & $35.4,45.7$ & 17.2 & 40.6 & $35.4,45.7$ & & 28.1 & $15.2,40.9$ & $p<.001$ \\
\hline Hagadera & & 39.0 & $37.4,40.6$ & 14.9 & 39.0 & $37.4,40.5$ & & 40.5 & $19.7,61.3$ & \\
\hline Ifo & & 41.7 & $39.7,43.8$ & 15.4 & 41.7 & $39.7,43.8$ & & 33.9 & $16.7,51.1$ & \\
\hline Kakuma & & 36.5 & $33.9,39.1$ & 14.3 & 35.5 & $32.6,38.3$ & & 43.6 & $38.8,48.4$ & $p<.01$ \\
\hline Rwanda & & 37.4 & $34.4,40.5$ & 20.1 & 37.4 & $34.4,40.5$ & 17.0 & - & & \\
\hline Gihembe & & 32.8 & $30.7,34.9$ & 17.3 & 32.8 & $30.7,34.9$ & & - & & \\
\hline Kiziba & & 38.6 & $34.2,43.0$ & 21.7 & 38.6 & $34.2,43.1$ & & 37.8 & $31.9,43.7$ & \\
\hline Nyabiheke & & 41.0 & $35.2,46.7$ & 21.4 & 41.0 & $35.2,46.7$ & & - & & \\
\hline Sudan & & 30.2 & $27.1,33.3$ & 9.2 & 27.1 & $25.2,29.0$ & 14.1 & 34.9 & $30.7,39.1$ & $\mathrm{p}<.001$ \\
\hline Abuda & & 27.6 & $22.9,32.3$ & 9.2 & 25.1 & $23.0,27.1$ & & 33.9 & $22.9,44.9$ & $p<.05$ \\
\hline Fau 5 & & 42.8 & $40.1,45.4$ & 9.5 & 34.4 & $31.3,37.5$ & & 47.8 & $44.0,51.6$ & $p<.001$ \\
\hline
\end{tabular}


Table 5 Percent of Outpatient Department Diagnoses by Children Less than Five Years of Age (U5), Refugee vs Host Country Patients, 2008-2009 (Continued)

\begin{tabular}{|c|c|c|c|c|c|c|c|c|c|}
\hline Girba & 29.6 & $27.3,31.9$ & 7.9 & 27.9 & $26.0,29.9$ & & 33.8 & $25.7,41.8$ & \\
\hline Kilo 26 & 18.9 & $16.5,21.3$ & 11.1 & 16.9 & $14.0,19.8$ & & 25.7 & $15.2,36.1$ & \\
\hline Shagarab | || ||| & 27.6 & $25.6,29.7$ & 14.8 & 27.3 & $25.4,29.1$ & & 29.5 & $20.7,38.2$ & \\
\hline Suki & 36.8 & $35.4,38.1$ & 4.5 & 31.9 & $28.3,35.4$ & & 39.6 & $35.6,43.5$ & \\
\hline Um Gargour & 29.7 & $27.1,32.3$ & 11.2 & 28.1 & $26.0,30.4$ & & 37.6 & $34.0,41.1$ & $p<.001$ \\
\hline Wad Sharifey & 30.0 & $25.3,34.7$ & 5.1 & 27.7 & $26.1,29.1$ & & 34.1 & $23.3,44.9$ & \\
\hline Tanzania & 41.8 & $38.1,45.4$ & 20.3 & 41.5 & $37.8,45.2$ & 17.8 & 44.2 & $42.1,46.2$ & $p<.05$ \\
\hline Lugufu & 50.0 & $47.7,52.2$ & 20.0 & 50.2 & $48.0,52.4$ & & 46.6 & $43.2,50.0$ & $p<.05$ \\
\hline Lukole & 43.8 & $39.3,48.2$ & 24.9 & 42.6 & $37.2,48.0$ & & 48.4 & $47.8,49.0$ & $p<.05$ \\
\hline Mtabila & 41.5 & $39.8,43.2$ & 20.0 & 41.3 & $39.5,43.0$ & & 44.0 & $42.9,46.9$ & $p<.01$ \\
\hline Nduta & 32.2 & $27 \cdot 0,37.3$ & 20.0 & 31.8 & $27.1,36.5$ & & 39.7 & $26.3,53.1$ & \\
\hline Nyarugusu & 39.6 & $38.3,40.9$ & 19.8 & 39.3 & $37.8,40.7$ & & 43.4 & $41.6,45.3$ & $p<.01$ \\
\hline Uganda & 37.8 & $36.4,39.2$ & 17.1 & 33.6 & $31.9,35.3$ & 19.5 & 40.8 & $38.9,42.8$ & $\mathrm{p}<.001$ \\
\hline Adjumani & 41.8 & $39.9,43.8$ & 14.3 & 35.2 & $34.0,36.4$ & & 44.7 & $42.3,47.2$ & $p<.001$ \\
\hline Ikafe & 45.8 & $42.8,48.7$ & 13.4 & 40.3 & $35.0,45.5$ & & 46.6 & $43.2,49.9$ & $p<.001$ \\
\hline Imvepi & 34.6 & $32.2,37.0$ & 10.9 & 24.1 & $21.6,26.7$ & & 41.0 & $35.6,46.4$ & $p<.001$ \\
\hline Kiryandongo & 36.3 & $34.4,38.3$ & 19.0 & 34.3 & $31.2,37.5$ & & 38.5 & $32.7,44.4$ & \\
\hline Kyaka II & 42.6 & $38.9,46.3$ & 24.8 & 41.3 & $37.4,45.3$ & & 45.3 & $39.7,50.8$ & \\
\hline Kyangwali & 38.9 & $36.2,41.5$ & 19.9 & 35.6 & $33.9,37.3$ & & 42.8 & $38.3,47.3$ & $p<.001$ \\
\hline Madi Okollo & 34.5 & $31.0,38.0$ & 15.7 & 30.0 & $26.5,33.4$ & & 37.8 & $30.7,44.9$ & $p<.001$ \\
\hline Nakivale & 31.6 & $27.7,35.6$ & 19.2 & 31.6 & $27.4,35.7$ & & 35.9 & $29.0,42.8$ & \\
\hline Oruchinga & 35.1 & $30.9,39.3$ & 21.5 & 30.9 & $26.1,35.7$ & & 36.7 & $30.3,43.2$ & \\
\hline Palorinya & 38.1 & $34.3,42.0$ & 15.1 & 38.3 & $35.2,41.5$ & & 38.5 & $33.0,44.0$ & \\
\hline Rhino & 38.7 & $32.2,45.2$ & 12.4 & 27.5 & $23.8,31.1$ & & 42.0 & $33.1,50.8$ & $p<.001$ \\
\hline Kharaz & 40.7 & $38.1,43.4$ & 19.7 & 40.4 & $36.7,44.2$ & 16.3 & 41.2 & $39.3,43.2$ & \\
\hline Zambia & 40.6 & $38.0,43.2$ & 19.7 & 40.8 & $38.3,43.3$ & 18.1 & 38.8 & $35.0,42.5$ & \\
\hline Kala & 40.9 & $38.5,43.4$ & 20.0 & 40.6 & $38.2,43.0$ & & 44.8 & $41.0,48.5$ & $p<.05$ \\
\hline Maheba & 39.4 & $35.6,43.2$ & 19.2 & 39.7 & $35.7,43.7$ & & 39.4 & $35.8,43.1$ & \\
\hline Mayukwayukwa & 36.1 & $34.2,38.0$ & 21.4 & 36.8 & $34.4,39.2$ & & 30.7 & $27.6,33.8$ & $p<.01$ \\
\hline Mwange & 46.9 & $41.5,52.4$ & 18.0 & 47.0 & $41.5,52.5$ & & 40.9 & $31.5,50.3$ & \\
\hline Asia & 30.0 & $28.9,31.1$ & 12.1 & 30.1 & $29.0,31.1$ & 9.8 & 24.4 & $21.5,27.2$ & $\mathrm{p}<.01$ \\
\hline Bangladesh & 34.8 & $32.9,36.7$ & 18.5 & 35.0 & $33.2,36.9$ & 10.4 & 22.8 & $15.1,30.5$ & $p<.01$ \\
\hline Kutupalong & 35.4 & $32.0,38.8$ & 19.0 & 35.5 & $32.2,38.9$ & & 23.7 & $15.0,32.4$ & $p<.01$ \\
\hline Nayapara & 34.1 & $32.3,35.9$ & 18.2 & 34.5 & $32.9,36.2$ & & 22.3 & $8.8,35.7$ & \\
\hline Nepal & 30.9 & $29.6,32.2$ & 8.0 & 30.8 & $29.4,32.1$ & 12.3 & 27.5 & $23.5,31.4$ & \\
\hline Beldangi I & 32.3 & $30.5,34.1$ & 8.6 & 32.3 & $30.5,34.1$ & & 30.8 & $23.6,38.0$ & \\
\hline Beldangi II & 28.6 & $26.3,30.9$ & 7.2 & 28.6 & $26.3,30.9$ & & 10.0 & $-3.3,23.4$ & $p<.01$ \\
\hline Beldangi II ext & 30.1 & $28.4,31.8$ & 8.1 & 30.1 & $28.4,31.8$ & & 18.2 & $8.7,27.7$ & $p<.01$ \\
\hline Goldhap & 32.6 & $29.7,35.4$ & 8.1 & 32.5 & $29.6,35.4$ & & 34.7 & $29.6,39.8$ & \\
\hline Khudunabari & 26.5 & $25.5,27.4$ & 6.8 & 25.7 & $24.7,26.8$ & & 38.7 & $33.5,44.0$ & $p<.001$ \\
\hline Sanishare & 36.3 & $34.7,38.0$ & 8.0 & 36.4 & $34.8,38.0$ & & 14.3 & $4.5,24.2$ & $p<.001$ \\
\hline Timai & 29.3 & $28.1,30.4$ & 8.7 & 29.1 & $27.9,30.3$ & & 32.4 & $26.9,37.8$ & \\
\hline Thailand & 28.2 & $26.6,29.6$ & 13.5 & 28.3 & $26.9,29.8$ & 7.2 & 18.4 & $15.4,21.5$ & $\mathrm{p}<.001$ \\
\hline Ban Don Yang & 24.7 & $23.8,25.7$ & 14.9 & 25.0 & $24.0,26.0$ & & 18.0 & $12.3,23.7$ & $p<.01$ \\
\hline Ban Mae Surin & 26.5 & $25.5,27.5$ & 13.8 & 26.5 & $25.5,27.5$ & & 8.3 & $-6.3,23.0$ & $p<.001$ \\
\hline $\begin{array}{l}\text { Ban Mai Nai } \\
\text { Soi }\end{array}$ & 39.2 & $35.9,42.6$ & 12.1 & 39.2 & $35.9,42.6$ & & - & & \\
\hline Mae La & 24.6 & $23.4,25.8$ & 11.1 & 24.8 & $23.6,26.0$ & & 16.1 & $9.0,23.2$ & $p<.001$ \\
\hline Mae La Oon & 28.7 & $26.9,30.4$ & 13.2 & 28.9 & $27.2,30.7$ & & 19.8 & $10.9,28.7$ & $p<.05$ \\
\hline $\begin{array}{l}\text { Mae Ra Ma } \\
\text { Luang }\end{array}$ & 26.4 & $25.3,27.5$ & 15.1 & 26.6 & $25.4,27.7$ & & 16.2 & $7.1,25.4$ & $p<.05$ \\
\hline Nu Poh & 26.0 & $25.1,26.9$ & 12.0 & 26.8 & $25.8,27.8$ & & 17.1 & $10.2,24.1$ & $p<.001$ \\
\hline
\end{tabular}


Table 5 Percent of Outpatient Department Diagnoses by Children Less than Five Years of Age (U5), Refugee vs Host Country Patients, 2008-2009 (Continued)

\begin{tabular}{|c|c|c|c|c|c|c|c|c|c|}
\hline Tham Hin & 30.5 & $28.7,32.4$ & 17.1 & 30.5 & $28.7,32.4$ & & 16.4 & $3.6,29.2$ & $p<.05$ \\
\hline Umpiem Mai & 25.9 & $24.8,27.0$ & 11.3 & 25.9 & $24.8,27.0$ & & 19.4 & $5.3,33.6$ & \\
\hline All Regions & 36.5 & $35.0,37.9$ & 15.7 & 35.6 & $34.7,36.6$ & 13.9 & 36.2 & $34.8,37.6$ & \\
\hline \multirow[t]{2}{*}{$\begin{array}{l}\text { Asia - Africa } \\
\text { Differential }\end{array}$} & -8.6 & $\begin{array}{c}-11.5,- \\
5.7\end{array}$ & -5.0 & -7.3 & $-9.3,-5.4$ & -6.4 & -15.0 & $\begin{array}{c}-17.8,- \\
12.3\end{array}$ & \\
\hline & & $p<.001$ & $p<.001$ & & $\mathrm{p}<.001$ & & & $p<.001$ & \\
\hline
\end{tabular}

* Values, Confidence Intervals and Significance are based on Generalized Estimating Equations, population-averaged model (Std. Err. adjusted for clustering on Camp); only p-values significant to the .05 level or less are provided.

** Source: World Bank, Health, Nutrition and Population database estimates for 2008 http://databank.worldbank.org.

Asia, it is 3.5. Both rates are within the range of 1-4 visits per person per year recommended by SPHERE for the emergency phase. The data in this analysis come from refugee settlements in the post-emergency phase, and therefore the SPHERE standard for emergencies may not be applicable, or may need to vary by region or context. The current SPHERE standard for emergencies of 1-4 visits per person per year appears to be relevant for Asia in the post-emergency phase, but not for Africa. In Africa, a post-emergency standard of 1-2 visits per person per year should be considered.

A few settlements had significant over-utilization rates ( $>4$ visits per person per year). One question is whether this increased utilization was due to a specific public health problem during the 2008-2009, or if it is due to specific cultural factors or health-seeking behaviors in certain populations. In contrast, some settlements had lower than expected utilization rates. This may suggest inadequate access to settlement health facilities, low quality of settlement health services, and/or the availability of competing health services of higher quality. It may also reflect acute events that restrict refugee access to health services in camps for limited periods. For example, insecurity (e.g. militia attacks in Chad) or natural disasters (e.g. local flooding in Kenya) or a mix may be explanations.

Analysis of gender differences in utilization rates reveals that female refugees utilize outpatient services at a higher rate (visits per person per year) than male refugees. This pattern of higher service utilization among female refugees is consistent across regions and countries. One possible explanation is that women use outpatient services for their own routine care, additional reproductive health needs, and are more likely than men to accompany children who need services [10].

\section{Distribution of Outpatient Service Users}

Overall, the number of refugees using settlement outpatient services is higher than the number of host nationals using the same services. This pattern is expected due to the remote/closed nature of refugee settlements in many countries. This means that-although in principle services are free of charge and accessible to nationals-host populations often prefer to visit host government sites closer by. UNHCR often also invests in local health services in refugee hosting areas (e.g., referral hospitals) which could help promote local access to them instead of services inside settlements. Other possible determinants of health service utilization are the direct and indirect costs of using the service and perceived quality of care [11]. However, the latter determinants are context specific and thus difficult to generalize for all refugee settlement situations.

In Uganda generally, and in some settlements in Sudan, however, the opposite trend is observed. In these special cases, host community members account for more visits to refugee settlement outpatient services than refugees. This may reflect the attention to integrated services for refugees and host nationals in Uganda, especially among settlements near the Sudanese border, that appears in the literature [4,12-14]. In Uganda, for example, refugee settlements are no longer refugee camps. Refugees were integrated into existing villages and health services, some of which already existed and others which were newly created and are available to all. The Ugandan Ministry of Health is now a direct implementing partner of UNHCR in some refugee settlements, and UNHCR entirely handed back services to local districts. No refugee-specific services exist anymore in these places, and therefore it is expected that refugee and host access will be more equitable.

In eastern Sudan, a number of refugee camps are located in remote areas more than $15 \mathrm{~km}$ from the nearest national health facility. Therefore, host populations living near to refugee camps prefer to seek care in the refugee health facilities, as they are much closer by walking distance (only $2-6 \mathrm{~km}$ ). Even in areas where national health facilities are available, refugee health facilities are often the preferred choice for host communities as there is a perception that national health services cannot meet the needs of host communities due to inadequate staffing and lack of basic medical supplies. In addition, high prescription and referral costs in national services often act as barriers to access to 
government services; whilst in comparison these tend to be more heavily subsidized within refugee camps.

The proportion of new OPD visits per month attributable to female refugees was higher than the female proportion of the refugee population (in all but one settlement). Similarly, in most African countries, the proportion of new OPD visits attributable to host national females was higher than national estimates of the proportion of females living in the host country. In Asia, in contrast, this happened only in Bangladesh. In Nepal and Thailand, females use refugee-settlement health services less than would be expected given their relative size of the population.

\section{Distribution of Diagnoses in Outpatient Services}

The proportion of outpatient diagnoses attributable to refugee children less than five years of age accounts for over one third (36\%) of all refugee outpatient diagnoses, despite the fact that the under five population makes up only $16 \%$ of the overall refugee population in this study. Very similar, although the national estimates of the size of the under-five population among host countries average at $14 \%$, under-fives also represent $36 \%$ of outpatient diagnoses among host nationals.

It is generally assumed that under-fives make up about $20 \%$ of the population in most emergency settings. In these protracted, post-emergency settings, however, it appears that the under-five population size approximates that of the host countries. For example, in Africa, under-fives represented $16-17 \%$ of both the refugee population and the national-level estimate for the host country. In Asia, under-fives represented 12\% of the refugee population, and $10 \%$ of the national estimate of the host country population. This is probably one explanation for why the proportion of all new outpatient diagnoses attributable to under-fives was lower in Asia (30\%) as compared to Africa (39\%).

The possible influences on the increased utilization among under-fives proportionate to population size are multi-factorial, such as the following: a child's nutritional status; the mother's knowledge and practice of how to prevent and appropriately manage childhood illness; the social and care environment of the household; and, increased susceptibility to infectious disease along with poor access to adequate water supply, sanitation, and immunizations. These are all potential factors leading to a larger number of diagnoses among these children compared to persons aged five years and above [15].

\section{Limitations}

Because we have no data about the size and distribution of the host populations that are using refugee settlement health facilities, we cannot assess the rate at which this population uses these settlement services. We are limited to observing the following among members of the host communities: (1) the percent of all visits made to the outpatient departments of refugee settlement facilities that are made by members of the host national community; (2) the proportion of these new outpatient visits by host nationals that are made by females vs. males; and (3) the proportion of new outpatient diagnoses by host nationals attributable to under-fives vs. those five years of age and older. The UNCHCR database disaggregates use of health services by only two age groups (under fives and five years and above). This limits how much we can identify differences in utilization by age. There may be variations between settlements in how utilization numbers and population numbers are collected and reported to UNHCR, making it difficult to ensure the validity of comparisons between settlements and countries. Finally, interpretation of the differences in specific settlements, countries and regions is somewhat limited by lack of contextual information in the database to explain these differences.

\section{Conclusions}

The availability of a centralized database of health information across UNHCR-supported refugee settlements is a rich resource that is only recently being utilized for across-settlement analyses. Several conclusions can be made from this initial analysis. As seen in Uganda, when refugee health services are integrated into existing host government services, refugees and locals clearly share these services more. This is good for equity but more work needs to be done to examine how quality of services change during and following integration.

The SPHERE standard for emergencies of 1-4 visits per person per year appears to be relevant for Asia in the post-emergency phase, but not for Africa. In Africa, a post-emergency standard of 1-2 visits per person per year should be considered, where investigation is indicated if the rate in particular settlement is above or below that standard. Why some settlements in the database had utilization rates higher or lower than the expected should be explored.

Although it is often assumed that the size of the female population in refugee settlements is higher than males, we found no statistically significant difference between the size of the male and female populations in refugee settlements overall. With a few exceptions, African settlements tended to have more females, whereas Asian settlements tended to have more males. The data do support the idea, however, that females utilize health services more than males and more than their representative size of the population.

Another assumption-that the under-fives make up $20 \%$ of the settlement population during the emergency 
phase-does not appear to hold for the post-emergency phase. Under-fives made up $17 \%$ of the refugee population in Africa, $12 \%$ of the population in Asian settlements, and $16 \%$ overall. Across both regions, under-fives use health services at a higher proportion than their numbers would suggest ( $37 \%$ of OPD visits vs. representing $16 \%$ of the population).

\section{Author details}

'Department of International Health, Johns Hopkins Bloomberg School of Public Health, 615 N. Wolfe Street, Baltimore, Maryland, 21205, USA.

2Department of Emergency Medicine, Johns Hopkins School of Medicine, Baltimore, Maryland 21205, USA. ${ }^{3}$ United Nations High Commissioner for Refugees, Geneva, Switzerland.

\section{Authors' contributions}

All authors have read and approved the final version of the manuscript. WW wrote key sections of the Methods, Results, Discussions and Conclusions. He also designed and carried out exploratory and statistical analysis. AV wrote key sections of the Background and Discussion and edited the manuscript. HT and SM compiled the data for analysis, helped write the Background, and edited the manuscript. CH and PS edited the manuscript and provided key input into the analysis approach and conclusions.

\section{Competing interests}

All authors have received salary support from the UN High Commissioner for Refugees. This salary support has covered implementation of the Health Information System described in this paper and/or for writing this manuscript.

Received: 6 June 2011 Accepted: 21 September 2011

Published: 21 September 2011

\section{References}

1. UNHCR: UNHCR 2008 Global Trends: refugees, asylum-seekers, returnees, internally displaced and stateless persons. June 2009. 2009.

2. CF PSpiegel, Colombo S, Palk E: Health-care needs of people affected by conflict: future trends and changing frameworks. Lancet 2010, 375(9711):341-345.

3. SM MHynes, Wilson HG, Spiegel P: Reproductive Health Indicators and Outcomes Among Refugee and Internally Displaced Persons in Postemergency Phase Camps. Journal of the American Medical Association 2002, 288:595-603.

4. DBV CGOrach: Postemergency health services for refugee and host populations in Uganda, 1999-2002. Lancet 2004, 364(9434):611-812.

5. Health Information System. [http://his.unhcr.org].

6. Health, Nutrition and Population Database. Demographic estimates for 2008. [http://databank.worldbank.org].

7. Tableau: Tableau Desktop Professional Edition Release 5.0 edition. Seattle, Wa.: Tableau Software; 2009.

8. StataCorp: Statistical Software: Release 11.0 edition. College Station, TX; 2009.

9. Project S: Sphere Handbook: Humanitarian Charter and Minimum Standards in Disaster Response. 2004, 268.

10. Rutta E, Williams H, Mwansasu A, Mung'ong'o F, Burke H, Gongo R, Veneranda R, Qassim M: Refugee perceptions of the quality of healthcare: findings from a participatory assessment in Ngara, Tanzania. Disasters 2005, 29(4):291-309.

11. Health WCotSDo: Closing the Gap in a Generation: Health Equity through Action on the Social Determinants of Health. 2008

12. Rowley EB, Gilbert, Drabe, Rabbin : Protracted Refugee Situations: Parallel Health Systems and Planning for the Integration of Services. 2006, 2:158-186, 19.

13. Burnham GMR, Elizabeth A, Ovberedjo, Martins O: Quality Design: A Planning Methodology for the Integration of Refugee and Local Health Services, West Nile, Uganda. Disasters 2003, 27(1):54-71.

14. Orach $\mathrm{CG}$, De Brouwere $\mathrm{V}$ : Integrating refugee and host health services in West Nile districts, Uganda. Health Policy Plan 2006, 21(1):53-64.
15. Young $H$, Borrel A, Holland D, Salama P: Public nutrition in complex emergencies. Lancet 2004, 364(9448):1899-1909.

doi:10.1186/1752-1505-5-19

Cite this article as: Weiss et al: Utilization of outpatient services in refugee settlement health facilities: a comparison by age, gender, and refugee versus host national status. Conflict and Health 2011 5:19.

\section{Submit your next manuscript to BioMed Central and take full advantage of:}

- Convenient online submission

- Thorough peer review

- No space constraints or color figure charges

- Immediate publication on acceptance

- Inclusion in PubMed, CAS, Scopus and Google Scholar

- Research which is freely available for redistribution

Submit your manuscript at www.biomedcentral.com/submit 